\title{
Malignant Adrenal Medulla Neoplasm
}

National Cancer Institute

\section{Source}

National Cancer Institute. Malignant Adrenal Medulla Neoplasm. NCI Thesaurus. Code C4396.

A primary or metastatic malignant neoplasm affecting the adrenal medulla. 